\title{
Generalized Köthe-Toeplitz Duals of Some Vector-Valued Sequence Spaces
}

\author{
Yilmaz Yilmaz \\ Department of Mathematics, Inonu University, 44280 Malatya, Turkey \\ Correspondence should be addressed to Y1lmaz Yılmaz; yyilmaz44@gmail.com
}

Received 17 August 2012; Accepted 26 November 2012

Academic Editor: Chuanxi Qian

Copyright (c) 2013 Yllmaz Yllmaz. This is an open access article distributed under the Creative Commons Attribution License, which permits unrestricted use, distribution, and reproduction in any medium, provided the original work is properly cited.

We know from the classical sequence spaces theory that there is a useful relationship between continuous and $\beta$-duals of a scalarvalued FK-space $E$ originated by the AK-property. Our main interest in this work is to expose relationships between the operator space $\mathscr{L}(E, Y)$ and $E^{\beta}$ and the generalized $\beta$-duals of some $X$-valued AK-space $E$ where $X$ and $Y$ are Banach spaces and $E^{\beta}=$ $\left\{\left(A_{k}\right), A_{k} \in \mathscr{L}(X, Y): \sum_{k=1}^{\infty} A_{k} x_{k}\right.$ converges in $Y$, for all $\left.x \in E\right\}$. Further, by these results, we obtain the generalized $\beta$-duals of some vector-valued Orlicz sequence spaces.

\section{Introduction}

The idea of dual sequence space was introduced by Köthe and Toeplitz [1]. Then, Maddox, [2], generalized this notion to $X$-valued sequence classes where $X$ is a Banach space. This brings an important contribution to the operator matrix transformation of Banach space-valued sequence spaces. Remember that $\beta$ - and $\alpha$-duals of a (complex-valued) sequence space $E$, denoted by $E^{\beta}$ and $E^{\alpha}$, respectively, are defined to be

$$
\begin{gathered}
E^{\beta}=\left\{\left(a_{k}\right) \in w: \sum_{k=1}^{\infty} a_{k} x_{k} \text { convergent for all } x=\left(x_{k}\right) \in E\right\}, \\
E^{\alpha}=\left\{\left(a_{k}\right) \in w: \sum_{k=1}^{\infty}\left|a_{k} x_{k}\right|<\infty \text { for all } x \in E\right\},
\end{gathered}
$$

where $w$ is the space of all complex-valued sequences. The $\left(a_{k}\right)$ in the classical definitions of Köthe-Toeplitz duals is replaced by a sequence $\left(A_{k}\right)_{k=1}^{\infty}$ of linear operators, not necessarily continuous, from $X$ into another Banach space $Y$. Thus, if $E$ is a nonempty set of sequences $x=\left(x_{k}\right)$ with $x_{k} \in X$, then generalized $\beta$ - and $\alpha$-duals of $E$ are defined to be

$$
\begin{gathered}
E^{\beta}=\left\{\left(A_{k}\right): \sum_{k=1}^{\infty} A_{k} x_{k} \text { convergent in the } Y\right. \text {-norm } \\
\text { for all } x \in E\}, \\
E^{\alpha}=\left\{\left(A_{k}\right): \sum_{k=1}^{\infty}\left\|A_{k} x_{k}\right\|<\infty \text { for all } x \in E\right\}
\end{gathered}
$$

respectively. It is clear that this notion depends on the space $Y$ and if $E \subset s(X)$, then

$$
E^{\alpha(\text { or } \beta)} \subset s(L(X, Y)),
$$

where $L(X, Y)$ is the space of all linear operators from $X$ into $Y$. Without the loss of generality we can restrict ourselves in this work to continuous operators and $\mathscr{L}(X, Y)$ being the space of all continuous linear operators from $X$ into $Y$ and $s(X)$ being the space of all $X$-valued sequences which is a natural generalization of $w=s(\mathbb{C})$.

We know from the classical sequence spaces theory that there is a useful relationship between continuous and $\beta$-duals of a sequence space whenever it has the AK-property. Related results are also expressed in [3, page 176]. Here, we are going 
to show that there is an analogue relationship for $X$-valued sequence spaces in the context of generalized $\beta$-duals with respect to another fixed Banach space $Y$. Further, by applying this result, we obtain generalized $\beta$-duals of some vectorvalued Orlicz sequence spaces. We think that our results give a fruitful way to find generalized duals of this kind of vectorvalued sequence spaces.

\section{Prerequisites}

We use the notations $\mathbb{N}, \mathbb{C}$, and $\mathbb{R}$ for the sets of all positive integers, complex numbers, and real numbers, respectively. For some locally convex (lc, for short) space $X, X^{*}$ denotes the continuous dual of $X$ and we denote by $B_{X}$ and $S_{X}$ the closed unit ball and the sphere of some normed space $X$, respectively.

An FH-space is an lc Fréchet space $E$ such that $E$ is a vector subspace of a Hausdorff topological vector space $H$ and the topology of $E$ is larger than the restricted topology of $H$ to $E$; that is, the inclusion map: $E \rightarrow H$ is continuous. If $H=w$ then an FH-space is called an FK-space. With a little extension, an $X$-valued sequence space $E$ is called an FK-space whenever $H=s(X)$ where $X$ is a Banach space. In fact, the theory of FK-spaces can be developed without the local convexity. However, we are interested only in locally convex FK-spaces. Note that, $s(X)=X^{\mathbb{N}}$ and so its topology is the weakest topology such that the projections

$$
P_{n}: s(X) \longrightarrow X, \quad P_{n}(x)=x_{n}, \quad n=1,2, \ldots
$$

are continuous.

An Orlicz function is a function $M:[0, \infty) \rightarrow[0, \infty)$ which is continuous, nondecreasing, and convex with $M(0)=$ $0, M(u)>0$ for all $u>0$ and $M(u) \rightarrow \infty$ as $u \rightarrow \infty$. An Orlicz function $M$ can always be represented in the following integral form:

$$
M(u)=\int_{0}^{u} p(t) d t
$$

where $p$, known as the kernel of $M$, is right differentiable for $t \geqslant 0, p(0)=0, p(t)>0$ for $t>0$, and $p$ is nondecreasing and $p(t) \rightarrow \infty$ as $t \rightarrow \infty$.

Consider the kernel $p(t)$ associated with Orlicz function $M(u)$, and let

$$
q(s)=\sup \{t: p(t) \leqslant s\}
$$

Then $q$ possesses the same properties as the function $p$. Suppose now

$$
N(v)=\int_{0}^{v} q(s) d s
$$

Then $N$ is an Orlicz function. The functions $M$ and $N$ are called mutually complementary Orlicz functions, and they satisfy the Young inequality,

$$
u v \leqslant M(u)+N(v) \text { for } u, v \geqslant 0 \text {. }
$$

An Orlicz function $M$ is said to satisfy the $\Delta_{2}$-condition for small $u$ at 0 if for each $k>0$ there exist $R_{k}>0$ and $u_{k}>0$ such that $M(k u) \leqslant R_{k} M(u)$, for all $u \in\left(0, u_{k}\right]$ [4]. that

The space $\ell_{M}$ consists of all sequences $\left(x_{k}\right)$ of scalars such

$$
\sum_{k=1}^{\infty} M\left(\frac{\left|x_{k}\right|}{\rho}\right)<\infty \text { for some } \rho>0,
$$

and it becomes a Banach space which is called an Orlicz sequence space with the Luxemburg norm

$$
\|x\|_{(M)}=\inf \left\{\rho>0: \sum_{k=1}^{\infty} M\left(\frac{\left|x_{k}\right|}{\rho}\right) \leqslant 1\right\} .
$$

The space $\ell_{M}$ is closely related to the space $\ell_{p}$ which is an Orlicz sequence space with $M(u)=u^{p},(1 \leqslant p<\infty)$.

Another definition of $\ell_{M}$, [4], is given by the complementary function to $M$ as follows:

$\ell_{M}=\left\{x=\left(x_{k}\right) \in w: \sum_{k=1}^{\infty} x_{k} y_{k}\right.$ converges, for all $\left.y \in \widetilde{\ell_{N}}\right\}$,

where $N$ is the complementary function to $M$ and $\widetilde{\ell_{N}}$ is the collection of all $x$ in $w$ with $\sum_{k=1}^{\infty} N\left(\left|x_{k}\right|\right)<\infty$. Clearly, $\widetilde{\ell_{N}} \subseteq$ $\ell_{N}$ and $\ell_{M}$ are normed by the Orlicz norm

$$
\|x\|_{M}=\sup \left\{\left|\sum_{k=1}^{\infty} x_{k} y_{k}\right|: \sum_{k=1}^{\infty} N\left(\left|y_{k}\right|\right) \leqslant 1\right\} .
$$

It was shown that these two norms on $\ell_{M}$ are equivalent.

An important closed subspace of $\ell_{M}$, introduced by $\mathrm{Y}$. Garibanov, is $h_{M}$ which is defined by

$h_{M}=\left\{x=\left(x_{k}\right) \in w: \sum_{k=1}^{\infty} M\left(\frac{\left|x_{k}\right|}{\rho}\right)<\infty\right.$, for all $\left.\rho>0\right\}$.

Immediately, we can introduce the vector-valued extension of the spaces $\ell_{M}$ and $h_{M}$ for any Banach space $X$. Therefore,

$$
\ell_{M}(X)=\left\{x \in s(X): \sum_{k=1}^{\infty} M\left(\frac{\left\|x_{k}\right\|}{\rho}\right)<\infty \text {, for some } \rho>0\right\},
$$

where $s(X)$ is the space of all $X$-valued sequences and $\|\cdot\|$ is the norm of $X . \ell_{M}(X)$ is a Banach space with the Luxemburg norm

$$
\|x\|_{(M)}=\inf \left\{\rho>0: \sum_{k=1}^{\infty} M\left(\frac{\left\|x_{k}\right\|}{\rho}\right) \leqslant 1\right\},
$$

and it coincides with $\ell_{M}$ whenever $X=\mathbb{C}$. Further, define the closed subspace $h_{M}(X)$ of $\ell_{M}(X)$ by $x=\left(x_{k}\right) \in h_{M}(X)$ if and only if

$$
\sum_{k=1}^{\infty} M\left(\frac{\left\|x_{k}\right\|}{\rho}\right)<\infty \quad \forall \rho>0 .
$$

If $M$ satisfies the $\Delta_{2}$-condition then $h_{M}(X)=\ell_{M}(X)$. 


\section{Relative Weak Topologies}

An operator $T \in \mathscr{L}(V, Y)$, for Banach spaces $V$ and $Y$, is called a Hahn-Banach operator if for every Banach space $X$ containing $V$ as a subspace there exists an operator $\widehat{T} \epsilon$ $\mathscr{L}(V, Y)$ such that $\|\widehat{T}\|=\|T\|$ and $\widehat{T} x=T x$, for every $x \in V$. Thus, the classical Hahn-Banach theorem can be restated in the following way for operators (see also [5]).

Theorem 1. Let $X, Y$ be Banach spaces and let $T: X \rightarrow Y$ be a continuous linear operator of rank 1 . Then $T$ is a HahnBanach operator.

By some modification on the assertion: norm preserving, the result remains true if $X$ is taken as a locally convex space. The evidence of this assertion can be found in [6, Section 7.2.].

Hence, by using Theorem 1 we derived some tools for later sections as in the way that is similar to classical treatments. The proof of the following result is also given in [7]. Nevertheless, it will be convenient to restate it here.

Corollary 2. Let $X, Y$ be Banach spaces and $x \in X$. Then, for some a $\in S_{Y}$, there exists a corresponding operator $T_{a}(\epsilon$ $\mathscr{L}(X, Y))$ such that

$$
\left\|T_{a}\right\|=1, \quad T_{a}(x)=\|x\| a .
$$

Proof. If $x$ is not null, take $V=\{\lambda x\}$. Then $V$ is a closed subspace of $X$ hence is a Banach space with the same norm. Define $T_{a}^{\prime}(\lambda x)=\lambda\|x\| a$, for some $a \in S_{Y}$, from $V$ into $Y$. Then $T_{a}^{\prime}$ satisfies the required condition of Theorem 1 on $V$. Hence it is a Hahn-Banach operator such that $\left\|T_{a}^{\prime}\right\|=1$. The norm preserving extension $T_{a}$ of $T_{a}^{\prime}$ to $X$ has the desired properties. The result is obvious for $x=0$.

Corollary 3. Let $X$ be an lc space, $Y$ be a Banach space, $S$ be a vector subspace of $X$, and $x \in X \backslash \bar{S}$. Then there exists an $a \in S_{Y}$ and a corresponding operator $T_{a}(\in L(X, Y))$ such that

$$
T_{a}(x)=a, \quad T_{a}=0 \text { on } S .
$$

Proof. Let $V=\{\lambda x\}$ and consider $Z=S \oplus V$. Fix some $a \in S_{Y}$ and define the operator

$$
T_{a}^{\prime}: Z \longrightarrow Y, \quad T_{a}^{\prime}(z)=T_{a}^{\prime}(s+\lambda x)=\lambda a,
$$

for $z \in Z$ (equivalently, for some $s \in S$ and $\lambda \in \mathbb{K}$ (= $\mathbb{C}$ or $\mathbb{R})$ such that $z=s+\lambda x)$. Clearly, the hyphothesis $x \in X \backslash \bar{S}$ says that $S$ is not dense in $Z$. Thus, $S$ must be closed in $Z$ since it is a maximal subspace of $Z$ (see [6, Prob. 4.2.5]). But $S=\operatorname{Ker} T_{a}^{\prime}=\{z \in Z: T(z)=0\}$, hence $T_{a}^{\prime}$ is continuous. Further, $T_{a}^{\prime}$ is an operator of rank 1 such that $T_{a}^{\prime}(x)=a$ and $T_{a}^{\prime}=0$ on $S$. Thus, the extension $T_{a}$ of $T_{a}^{\prime}$ is the desired operator.

Let us establish an lc topology on a Banach space $X$ with respect to another Banach space $Y$. Let $w_{Y}$ be a topology on $X$ such that, for each net $x=\left(x_{\delta}\right)$ in $X, x_{\delta} \rightarrow 0\left(w_{Y}\right)$ if and only if $\left\|T\left(x_{\delta}\right)\right\|_{Y} \rightarrow 0$ for each $T \in \mathscr{L}(X, Y)$. It is an lc topology generated by the family $P_{Y}=\left\{\|\cdot\|_{Y} \circ T: T \in \mathscr{L}(X, Y)\right\}$ of the seminorms $\|\cdot\|_{Y} \circ T$ on $X$. Obviously, the norm topology of $X$ is stronger than $w_{Y}$, in general. If $Y$ is a scalar field of $X$ then $w_{Y}$ coincide with the usual weak topology. It is clear that, a net which is $w_{Y}$-convergent to 0 is also weak convergent to 0 . The converse of this assertion is not true.

Example 4. Let $X=Y=\ell_{2}$. Then the sequence $\left(e_{n}\right)_{n=1}^{\infty}$ of unit vectors is weak convergent to 0 in $\ell_{2}$ [8, page 99]. But, it is not $w_{\ell_{2}}$-convergent to 0 . Therefore, for the identity operator on $\ell_{2}$, we have $\left\|I e_{n}\right\|=\left\|e_{n}\right\|=1 \nrightarrow 0$.

However, we cannot work this example in $\ell_{1}$ (in fact, in a Banach space which has the Schur property) since weak convergence implies the norm convergence in this case. Hence the following result is obvious from the definition of the Schur property.

Theorem 5. Let $X$ be a Banach space having the Schur property. Then weak convergence implies $w_{Y}$-convergence in $X$ for every Banach space $Y$.

Now consider the canonical embedding $X \rightarrow \mathscr{L}^{2}(X, Y)$, where $\mathscr{L}^{2}(X, Y)$ is the space of all continuous operators from $\mathscr{L}(X, Y)$ into $Y$ and $X$ and $Y$ are Banach spaces, which assigns each $x \in X$ to the operator $F_{x}$ on $\mathscr{L}(X, Y)$ defined by

$$
F_{x}(T)=T x, \quad \text { for each } T \in \mathscr{L}(X, Y) \text {. }
$$

Clearly,

$$
\left\|F_{x}(T)\right\|_{Y}=\|T x\|_{Y} \leq\|T\|\|x\|_{X}
$$

so that $F_{x} \in \mathscr{L}^{2}(X, Y)$ and

$$
\left\|F_{x}\right\| \leq\|x\|_{X} .
$$

Theorem 1 and the succeeding corollary assert that the canonical embedding is a linear isometry from $X$ into $\mathscr{L}^{2}(X, Y)$ as is in the classical case.

Now, let us investigate how do the bounded subsets of the $X$ in the $w_{Y}$-topology behave. Note that a subset $A$ of $X$ is called $w_{Y}$-bounded if $T(A)$ is bounded in $Y$ for each $T \in \mathscr{L}(X, Y)$. It is clear that, for every pair of the Banach spaces $X$ and $Y, A \subseteq X$ is $w_{Y}$-bounded if it is norm bounded. The converse of this assertion is the following theorem.

Theorem 6. Let $X$ and $Y$ be Banach spaces. Then $w_{Y}$-bounded sets are norm bounded.

Proof. Let $V \subset X$ be $w_{Y}$-bounded and $\widehat{V}$ be canonical embedding of $V$ into $\mathscr{L}^{2}(X, Y)$. A hypothesis says that $\widehat{V}$ is pointwise bounded so it is uniformly (norm) bounded by the uniform boundedness principle. Hence there exists a $K>0$ such that $\left\|F_{x}\right\| \leq K$ for each $x \in V$. So

$$
\|x\|=\left\|F_{x}\right\| \leq K
$$

for each $x \in V$. 
Theorem 7. Let $X$ be an lc space and $Y$ be a Banach space. Then $w_{Y}$-bounded sets are also bounded in the lc topology of $X$.

Proof. Let $V \subset X$ be $w_{Y}$-bounded. We are going to show that $p[V]$ is bounded for each seminorm $p$ in $P$ where $P$ is the family of all seminorms generating the lc topology of $X$. For an arbitrary $p, Z=(X, p)$ is a seminormed space. Thus we can show as in Theorem 6 that $\widehat{V}$ is bounded in $\mathscr{L}^{2}(Z, Y)$, whence, $V$ is bounded in $Z$, that is, $p[V]$ is a bounded subset of $\mathbb{R}$.

We conclude this section with a brief discussion of equicontinuity. A set $\Omega$ of linear maps from one topological vector space $X$ into another one $Y$ is called equicontinuous if, for each neighborhood $N$ of 0 in $Y$,

$$
\bigcap\left\{T^{-1}[N]: T \in \Omega\right\}
$$

is a neighborhood of 0 in $X$. Equicontinuity is a generalization of the uniform boundedness of the family of linear maps between seminormed spaces.

Theorem 8 (see [9]). Let $\Omega$ be a collection of continuous linear mappings $T$ from the Fréchet space $X$ into the topological vector space $Y$. Then $\Omega$ is equicontinuous if and only if the set

$$
\Omega(x)=\{T x: T \in \Omega\}
$$

is bounded in $Y$, for each $x \in X$.

Lemma 9 (see [6]). Let $\left(T_{\delta}\right)$ be a net of continuous operators $T_{\delta}$ from a Fréchet space $X$ into the topological vector space $Y$. Then the set $\left\{x: T_{\delta} x \rightarrow 0\right\}$ is a closed subspace of $X$.

\section{Sectional Properties and Operator Spaces}

For an $x \in s(X)$,

$$
x^{(n)}=\left(x_{1}, x_{2}, \ldots, x_{n}, 0, \ldots\right)
$$

is called $n$th section of $x$. Further, $\phi(X)$ denotes the space of all finite sequences in $s(X)$.

Definition 10. Let $E \supset \phi(X)$ be an FK-space. If, for each $x \in$ E,

$$
x^{(n)} \longrightarrow x \text { in } E \text {, }
$$

then $E$ is called an AK-space. Further, $E$ is called an AD-space whenever $\phi(X)$ is dense in $E$. If, for each $x \in E$, the sequence $\left(x^{(n)}\right)$ is bounded in $E$ then $E$ is called an AB-space.

An AK-(AD-, AB-) space is also called to have AK-(AD-, AB-) property.

Let $E \supset \phi(X)$ be an FK-space and define the set

$$
B=\left\{x \in E:\left(x^{(n)}\right) \text { is bounded in } E\right\} .
$$

Clearly, $B \subset E$ and $B=E$ whenever $E$ is an AB-space.
To define another important classes we consider the mappings

$$
I_{k}: X \longrightarrow E \quad I_{k}(a)=(0, \ldots, 0, \stackrel{k \text { th position }}{a}, 0, \ldots)
$$

and define the set $W_{Y}$, for some Banach space $Y$, by

$$
W_{Y}=\left\{x \in E: T x=\sum\left(T \circ I_{k}\right)\left(x_{k}\right) \forall T \in \mathscr{L}(E, Y)\right\} .
$$

Proposition 11. For each Banach space $Y, W_{Y} \subset B$.

Proof. Let $x \in W_{Y}$ and $T \in \mathscr{L}(E, Y)$ then we can write $T x=$ $\sum\left(T \circ I_{k}\right)\left(x_{k}\right)$, that is,

$$
\sum_{k=1}^{n}\left(T \circ I_{k}\right)\left(x_{k}\right) \longrightarrow T x(n \longrightarrow \infty) \text { in } E \text {. }
$$

Since

$$
\sum_{k=1}^{n}\left(T \circ I_{k}\right)\left(x_{k}\right)=T x^{(n)}
$$

$T x^{(n)} \rightarrow T x$; that is, the sequence $\left\{x^{(n)}\right\}$ is $w_{Y}$-convergent hence it is $w_{Y}$-bounded. Thus, it is also bounded in the lc Fréchet topology of $E$ by Theorem 7.

Proposition 12. For each Banach space $Y$,

$$
W_{Y} \subset \overline{\phi(X)}
$$

where $\overline{\phi(X)}$ is the closure of $\phi(X)$ in $E$.

Proof. Let $x \in W_{Y}$. Then, for every $T \in \mathscr{L}(E, Y)$ such that $T=0$ on $\phi(X)$,

$$
\begin{aligned}
T x & =\sum\left(T \circ I_{k}\right)\left(x_{k}\right)=\sum T(0, \ldots, 0, \stackrel{k \text { th }}{x}, 0, \ldots) \\
& =\sum 0=0 .
\end{aligned}
$$

This implies $x \in \overline{\phi(X)}$. If this is not so, then there exists an $a \in S_{Y}$ and a corresponding operator $T_{a}(\in \mathscr{L}(E, Y))$ such that

$$
T_{a}(x)=a \neq 0, \quad T_{a}=0 \text { on } \phi(X)
$$

by Corollary 3 . This is a contradiction.

Proposition 13. Let $E \supset \phi(X)$ be an FK-space with $A D$ - and $A B$-property. Then $E$ also has the AK-property.

Proof. Define

$$
A_{n}: E \longrightarrow E, \quad A_{n}(x)=x^{(n)}-x, \quad \text { for } n=1,2, \ldots
$$

Then the sequence $\left\{A_{n}(x)\right\}$ is bounded by the AB-property. Therefore $\left\{A_{n}\right\}$ is equicontinuous by Theorem 8 . On the other hand $A_{n}(x) \rightarrow 0$ for each $x \in \phi(X)$, That is,

$$
\phi(X) \subset\left\{x \in E ; A_{n}(x) \longrightarrow 0\right\}=\Lambda .
$$


Since $\Lambda$ is a closed subspace of $E$ from Lemma 9, we obtain that $\overline{\phi(X)} \subseteq \Lambda$. Thus

$$
A_{n}(x)=x^{(n)}-x \longrightarrow 0
$$

for each $x \in \overline{\phi(X)}$ (=E by the AD-property), whence, $E$ has the AK-property.

Theorem 14. Let $E \supset \phi(X)$ be an FK-space. Then $E$ is an AKspace if and only if $E^{\beta}$ and $\mathscr{L}(E, Y)$ are isomorphic for every Banach space $Y$.

Proof. Let $\left(T_{k}\right) \in E^{\beta}$ where each $T_{k} \in \mathscr{L}(X, Y)$ and define $A\left[\left(T_{k}\right)\right]=T$ by

$$
T x=\sum_{k=1}^{\infty} T_{k} x_{k}
$$

for each $x \in E$. Write

$$
\eta_{n}=\sum_{k=1}^{n} T_{k} \circ P_{k}
$$

where $P_{k}: E \rightarrow X, k=1,2, \ldots$ is the $k$ th (continuous) projection defined by $P_{k}(x)=x_{k}$. Then each $\eta_{n}$ is continuous and the sequence $\left(\eta_{n}\right)$ is pointwise convergent since the series $\sum T_{k} x_{k}$ is convergent. So, the operator $T$, which is also defined by

$$
T x=\lim \eta_{n}(x),
$$

is continuous by the Banach-Steinhauss closure theorem, whence, $T \in \mathscr{L}(E, Y)$. That $A$ is injective comes from the following discussion. Let $A\left[\left(T_{k}\right)\right]=T=0$. Then, for each $a \in X$,

$$
\left(T \circ I_{k}\right)(a)=T_{k} a=0 .
$$

This implies each $T_{k}=0$, that is, $\left(T_{k}\right)=0$. Further, for each $T \in \mathscr{L}(E, Y)$, let us consider

$$
T_{k}=T \circ I_{k}, \quad \text { for } k=1,2, \ldots,
$$

from $X$ to $Y$. For each $x \in E$,

$$
\sum_{k=1}^{n} T_{k} x_{k}=\sum_{k=1}^{n}\left(T \circ I_{k}\right)\left(x_{k}\right) \longrightarrow T x \quad(n \longrightarrow \infty)
$$

since $E$ is an AK-space, whence, $\left(T_{k}\right) \in E^{\beta}$. This means that $A$ is surjective.

Conversely, let $E^{\beta}$ and $\mathscr{L}(E, Y)$ be isomorphic. Then each $T \in \mathscr{L}(E, Y)$ has the representation $\left(T_{k}\right)$ such that each

$$
T_{k}=T \circ I_{k} \in \mathscr{L}(E, Y)
$$

and also, for each $x \in E$,

$$
T x=\sum T_{k} x_{k}
$$

This shows that $x \in W_{Y}$, that is,

$$
E \subseteq W_{Y} .
$$

Thus, we obtain $E=\overline{\phi(X)}$ by the Proposition 12, whence, $E$ has the AD-property. Also, $E$ has the AB-property by Proposition 11. Hence, $E$ is an AK-space by Proposition 13.

\section{Applications on Vector-Valued Orlicz Sequence Spaces}

It is not hard to see as in the classical case, [4], that another definition of $\ell_{M}(X)$ by the complementary function $N$ to $M$ is

$$
\begin{gathered}
\ell_{M}(X)=\left\{x \in s(X): \sum_{k=1}^{\infty} f_{k}\left(x_{k}\right)\right. \text { converges, } \\
\text { for all } \left.f=\left(f_{k}\right) \in \widetilde{\ell_{N}}\left(X^{*}\right)\right\},
\end{gathered}
$$

where $\widetilde{\ell_{N}}\left(X^{*}\right)$ is the class of all sequences $f=\left(f_{k}\right)$ such that $\sum_{k=1}^{\infty} N\left(\left\|f_{k}\right\|\right)<\infty$ and each $f_{k} \in X^{*}$. Further, for each $x \in$ $\ell_{M}(X)$,

$$
\|x\|_{M}=\sup \left\{\left|\sum_{k=1}^{\infty} f_{k}\left(x_{k}\right)\right|: \sum_{k=1}^{\infty} N\left(\left\|f_{k}\right\|\right) \leqslant 1\right\}<\infty
$$

defines a norm on $\ell_{M}(X)$. This norm is said to be Orlicz norm on $\ell_{M}(X)$.

Lemma 15. On $\ell_{M}(X)$, the norms $\|\cdot\|_{M}$ and $\|\cdot\|_{(M)}$ are equivalent, and $\|x\|_{(M)} \leqslant\|x\|_{M} \leqslant 2\|x\|_{(M)}$.

Proofs of this lemma and the above assertion can be given in a similar way followed in [4, Theorem 8.9], by using the inequality

$$
\left|\sum_{k=1}^{\infty} f_{k}\left(x_{k}\right)\right| \leqslant \sum_{k=1}^{\infty}\left\|f_{k}\right\|\left\|x_{k}\right\|
$$

and by using the fact that $x=\left(x_{k}\right) \in \ell_{M}(X)$ if and only if $\left(\left\|x_{k}\right\|\right)_{k=1}^{\infty} \in \ell_{M}$.

Lemma 16. Let $M$ be an Orlicz function. The sets

$$
\begin{aligned}
& \Lambda_{1}=\left\{x \in s(X): \sum_{k=1}^{\infty} M\left(\left\|x_{k}\right\|\right) \leqslant 1\right\}, \\
& \Lambda_{2}=\left\{x \in s(X):\|x\|_{(M)} \leqslant 1\right\}
\end{aligned}
$$

are identical.

Proof. Let $x \in \Lambda_{1}$, this means $\sum_{k=1}^{\infty} M\left(\left\|x_{k}\right\| / \rho\right) \leqslant 1$ for $\rho=1$. Hence, $\|x\|_{(M)} \leqslant 1$, that is, $x \in \Lambda_{2}$. Conversely, let $x \in \Lambda_{2}$, that is

$$
\inf \left\{\rho>0: \sum_{k=1}^{\infty} M\left(\frac{\left\|x_{k}\right\|}{\rho}\right) \leqslant 1\right\} \leqslant 1
$$

This means $\sum_{k=1}^{\infty} M\left(\left\|x_{k}\right\| / \rho\right) \leqslant 1$ for some $\rho \leqslant 1$. Therefore $\sum_{k=1}^{\infty} M\left(\left\|x_{k}\right\|\right) \leqslant 1$ since $M$ is nondecreasing.

In general $h_{M}(X)$ has no Schauder basis in classical manner. In [10] we introduce a new kind basis notion. Let us give this definition and prove that $h_{M}(X)$ has a basis in this manner. 
Definition 17 (see [10]). Let $X$ and $Y$ be Banach spaces and $\mathbb{A}$ be a set. A family $\left\{\eta_{a}: a \in \mathbb{A}\right\}$ of continuous linear functions $\eta_{a}: Y \rightarrow X$ is called $Y$-basis for $X$ if the following condition is satisfied. There exists a directed subset $\mathscr{D}$ (by some relation $\ll)$ of $\mathscr{F}$ satisfying the property; for each $a \in \mathbb{A}$ there is some $F \in \mathscr{D}$ such that $a \in F$, and there exists a unique family $\left\{R_{a}: a \in \mathbb{A}\right\}$ of linear functions $R_{a}$ from $X$ onto $Y$ such that, for each $x \in X$, the net $\left(\pi_{F}(x): \mathscr{D}, \ll\right)$ converges to $x$ in $X$ where

$$
\pi_{F}(x)=\sum_{a \in F}\left(\eta_{a} \circ R_{a}\right)(x),
$$

for each $F \in \mathscr{D}$ and $\mathscr{F}$ is the family of all finite subsets of the index set $\mathbb{A}$ which is directed by the inclusion relation $\subseteq$. Furthermore, $\left\{\eta_{a}\right\}$ is called a $Y$-Schauder basis for $X$ whenever each $R_{a}$ is continuous.

Thus we say that each $x \in X$ has the representation

$$
x=\sum_{a \in \mathbb{A}}\left(\eta_{a} \circ R_{a}\right)(x),
$$

in this case.

Definition 18. The $Y$-basis $\left\{\eta_{a}: a \in \mathbb{A}\right\}$ in the above definition is called unconditional whenever $\mathscr{D}=\mathscr{F}$ with the inclusion relation $\subseteq$.

By taking $\mathbb{A}=\mathbb{N}$ in the Definition [10] we now prove that $h_{M}(X)$ has an unconditional $X$-Schauder basis.

Theorem 19. For $k \in \mathbb{N}$ consider again the operators $I_{k}$ : $X \rightarrow h_{M}(X)$ such that

$$
I_{k}(u)=(0,0, \ldots, 0, \stackrel{\text { kthposition }}{u}, 0, \ldots)
$$

Then, the sequence $\left\{I_{k}\right\}$ is an unconditional X-Schauder basis for $h_{M}(X)$.

Proof. Let us take $R_{k}=P_{k}: h_{M}(X) \rightarrow X, P_{k}(x)=x_{k}$ as a coordinate projection in the Definition [10]. We should prove that the net $\left(\pi_{F}(x): \mathscr{F}, \subseteq\right)$ converges to $x$ in $h_{M}(X)$. This means, for each $\epsilon>0$, we should find an $F_{0} \in \mathscr{F}$ such that $\left\|x-\pi_{F}(x)\right\|_{(M)}<\epsilon$ for $F_{0} \subseteq F$. Now, let $\epsilon>0$ be given. Since $\sum_{k=1}^{\infty} M\left(\left\|x_{k}\right\| / \rho\right)<\infty$ for every $\rho>0$, especially for $\epsilon>0$, the series $\sum_{k=1}^{\infty} M\left(\left\|x_{k}\right\| / \epsilon\right)$ is absolutely convergent and hence it is unconditional convergent in real numbers. Hence we can find an $n_{0}(\epsilon)$ such that $\sum_{k=n_{0}+1}^{\infty} M\left(\left\|x_{k}\right\| / \epsilon\right) \leq 1$. Now let $F_{0}=\left\{1,2, \ldots, n_{0}\right\}$. Obviously, $F_{0}$ is dependent on $\epsilon$ and the set

$$
\left\{\rho>0: \sum_{k \in \mathbb{N} \backslash F_{0}} M\left(\frac{\left\|x_{k}\right\|}{\rho}\right) \leqslant 1\right\}
$$

includes the $\epsilon$. This means

$$
\inf \left\{\rho>0 \sum_{k \in \mathbb{N} \backslash F} M\left(\frac{\left\|x_{k}\right\|}{\rho}\right) \leqslant 1\right\} \leqslant \epsilon .
$$

Now, remember that

$$
\pi_{F}(x)=\sum_{k \in F}\left(I_{k} \circ P_{k}\right)(x) .
$$

Hence, for some $F \in \mathscr{F}$ such that $F_{0} \subseteq F$, we have

$$
\begin{aligned}
\| x- & \pi_{F}(x) \|_{(M)} \\
= & \|\left(x_{1}, \ldots, x_{n_{1}-1}, 0, x_{n_{1}+1} \ldots x_{n_{2}-1}, 0,\right. \\
& \left.\quad x_{n_{2}+1}, \ldots x_{n_{m}-1}, 0, x_{n_{m}+1} \ldots\right) \|_{(M)} \\
= & \inf \left\{\rho>0: \sum_{k \in \mathbb{N} \backslash F} M\left(\frac{\left\|x_{k}\right\|}{\rho}\right) \leqslant 1\right\} \\
\leqslant & \epsilon .
\end{aligned}
$$

The continuity of each $P_{k}$ and uniqueness of the sequence $\left\{P_{k}\right\}$ in the representation can be done similarly in the classical case. This completes the proof.

One of our main results is the following theorem which states the generalized $\beta$-dual of $h_{M}(X)$ with respect to the Banach space $Y$. The above theorem brings that $h_{M}(X)$ is an AK-space and we can use Theorem 14 to find the generalized $\beta$-dual of $h_{M}(X)$

Theorem 20. Let $X, Y$ be Banach spaces and $M, N$ be mutually complementary Orlicz functions. Then, $h_{M}(X)^{\beta}$ is isomorphic by the mapping $T \rightarrow\left(T \circ I_{k}\right)$ to the Banach space

$$
\begin{aligned}
V_{N}=\left\{A=\left(A_{k}\right) \in s(\mathscr{B}(X, Y)),\right. \\
\left.\|A\|=\sup _{f \in B_{Y^{*}}}\left\|\left(A_{k}^{*} f\right)_{k=1}^{\infty}\right\|_{N}<\infty\right\},
\end{aligned}
$$

where each $I_{k}$ is defined as in Theorem 19.

Proof. We prove that $\mathscr{L}\left(h_{M}(X), Y\right)$ is isometrically isomorphic to $V_{N}$.

$\mathrm{A}$ routine calculation shows that $\|A\|=$ $\sup _{f \in B_{Y^{*}}}\left\|\left(A_{k}^{*} f\right)_{k=1}^{\infty}\right\|_{N}$ really defines a norm on $V_{N}$ and it is a Banach space with this norm. Let $T \in \mathscr{L}\left(h_{M}(X), Y\right)$ and say $A_{k}=T \circ I_{k}$ for each $k$. This implies $\left\|\left(A_{k} \circ P_{k}\right)(x)\right\|=0$ so that $A_{k}\left(x_{k}\right)=0$ for each $k$. Since each $x \in h_{M}(X)$ has the unconditional representation $x=\sum_{k=1}^{\infty}\left(I_{k} \circ P_{k}\right)(x)$, we can write

$$
T x=\sum_{k=1}^{\infty}\left(T \circ I_{k}\right)\left(x_{k}\right)=\sum_{k=1}^{\infty} A_{k} x_{k} .
$$

Immediately each $A_{k} \in \mathscr{L}(X, Y)$ since $\left\|A_{k}\right\| \leqslant\|T\|\left\|I_{k}\right\|=$ $\|T\|$. Now, let us define the mapping

$$
\begin{gathered}
\Psi: \mathscr{L}\left(h_{M}(X), Y\right) \longrightarrow V_{N}, \\
\text { by } \Psi(T)=A=\left(A_{k}\right)_{k=1}^{\infty} ; \quad A_{k}=T \circ I_{k} .
\end{gathered}
$$


$\Psi(T)=0$ if and only if each $T \circ I_{k}=0$ so $T=0$ by the definition of each $I_{k}$, that is, $\Psi$ is one to one. Also, for an arbitrary $A \in V_{N}$, if we define the operator $T$ by

$$
T x=\sum_{k=1}^{\infty} A_{k} x_{k}
$$

on $h_{M}(X)$ then, by using the Young inequality, we have

$$
\begin{aligned}
\left\|\sum_{k=m}^{n} A_{k} x_{k}\right\|= & \sup _{f \in B_{Y^{*}}}\left|f\left(\sum_{k=m}^{n} A_{k} x_{k}\right)\right| \\
\leqslant & \sup _{f \in B_{Y^{*}}} \sum_{k=m}^{n}\left\|A_{k}^{*} f\right\|\left\|x_{k}\right\| \\
\leqslant & \sup _{f \in B_{Y^{*}}} \sum_{k=m}^{n} N\left(\frac{\left\|A_{k}^{*} f\right\|}{\left\|\left(A_{k}^{*} f\right)_{k=1}^{\infty}\right\|_{N}}\right) \\
& +\sup _{f \in B_{Y^{*}}} \sum_{k=m}^{n} M\left(\left\|\left(A_{k}^{*} f\right)_{k=1}^{\infty}\right\|_{N}\left\|x_{k}\right\|\right) \\
\leqslant & \sup _{f \in B_{Y^{*}}} \sum_{k=m}^{n} N\left(\frac{\left\|A_{k}^{*} f\right\|}{\left\|\left(A_{k}^{*} f\right)_{k=1}^{\infty}\right\|_{N}}\right) \\
& +\sum_{k=m}^{n} M\left(\frac{\left\|x_{k}\right\|}{1 /\|A\|}\right) .
\end{aligned}
$$

Since $\left(A_{k}^{*} f\right)_{k=1}^{\infty} \in \ell_{N}(\mathscr{L}(X, Y))$ for each $f \in Y^{*}$ and

$$
\sum_{k} N\left(\frac{\left\|A_{k}^{*} f\right\|}{\left\|\left(A_{k}^{*} f\right)_{k=1}^{\infty}\right\|_{N}}\right) \leqslant 1
$$

from [4, Prop. 8.12], we have

$$
\sup _{f \in B_{Y^{*}}} \sum_{k=m}^{n} N\left(\frac{\left\|A_{k}^{*} f\right\|}{\left\|\left(A_{k}^{*} f\right)_{k=1}^{\infty}\right\|_{N}}\right) \longrightarrow 0 \quad \text { as } m, n \longrightarrow \infty \text {. }
$$

Also

$$
\sum_{k=m}^{n} M\left(\frac{\left\|x_{k}\right\|}{1 /\|A\|}\right) \longrightarrow 0 \text { as } m, n \longrightarrow \infty
$$

since $x \in h_{M}(X)$. This means the series $\sum A_{k}\left(x_{k}\right)$ is convergent, that is, $T$ is well defined. Further, that the mapping $\Psi$ is onto, that is, $T \in \mathscr{B}\left(h_{M}(X), Y\right)$ comes from the following equalities:

$$
\begin{aligned}
\|T\|= & \sup _{x \in B_{M}}\|T x\|=\sup _{x \in B_{M}}|| \sum_{k=1}^{\infty} A_{k}\left(x_{k}\right) \| \\
= & \sup _{x \in B_{M}} \sup _{f \in B_{Y^{*}}}\left|f\left(\sum_{k=1}^{\infty} A_{k}\left(x_{k}\right)\right)\right| \\
= & \sup _{f \in B_{Y^{*}}} \sup _{x \in B_{M}}\left|\sum_{k=1}^{\infty}\left(A_{k}^{*} f\right)\left(x_{k}\right)\right| \\
= & \sup _{f \in B_{Y^{*}}} \sup \left\{\left|\sum_{k=1}^{\infty}\left(A_{k}^{*} f\right)\left(x_{k}\right)\right|: \sum_{k=1}^{\infty} M\left(\left\|x_{k}\right\|\right) \leqslant 1\right\}, \\
& (\operatorname{by} \operatorname{Lemma} 16) \\
= & \sup _{f \in B_{Y^{*}}}\left\|\left(A_{k}^{*} f\right)_{k=1}^{\infty}\right\|_{N}=\|A\| .
\end{aligned}
$$

This shows at the same time that $\Psi$ is an isometry.

\section{References}

[1] G. Köthe and O. Toeplitz, "Lineare räume mit unendlich vielen koordinaten und reigne unendlicher matrizen, jour," Journal für die Reine und Angewandte Mathematik, vol. 171, pp. 193-226, 1934.

[2] I. J. Maddox, Infinite Matrices of Operators, Springer, Heidelberg, Germany, 1980.

[3] A. Wilansky, Summability Through Functional Analysis, NorthHolland Mathematics Studies, Amsterdam, The Netherlands, 1984.

[4] P. K. Kamthan and M. Gupta, Sequence Spaces and Series, Marcel Dekker, New York, NY, USA, 1981.

[5] M. I. Ostrovskii, "Hahn-Banach operators," Proceedings of the American Mathematical Society, vol. 129, no. 10, pp. 2923-2930, 2001.

[6] A. Wilansky, Modern Methods in Topological Vector Spaces, McGraw Hill, New York, NY, USA, 1978.

[7] Y. Yllmaz, "Characterizations of some operator spaces by relative adjoint operators," Nonlinear Analysis: Theory, Methods and Applications, vol. 65, no. 10, pp. 1833-1842, 2006.

[8] B. Choudhary and S. Nanda, Functional Analysis with Applications, John Wiley \& Sons, New York, NY, USA, 1989.

[9] G. Köthe, Topological Vector Spaces, Springer, Heidelberg, Germany, 1969.

[10] Y. Y1lmaz, "Relative bases in Banach spaces," Nonlinear Analysis: Theory, Methods and Applications, vol. 71, no. 5-6, pp. 2012-2021, 2009. 


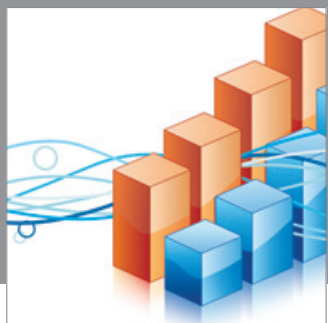

Advances in

Operations Research

mansans

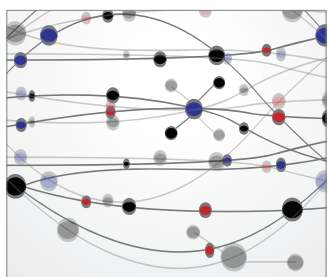

The Scientific World Journal
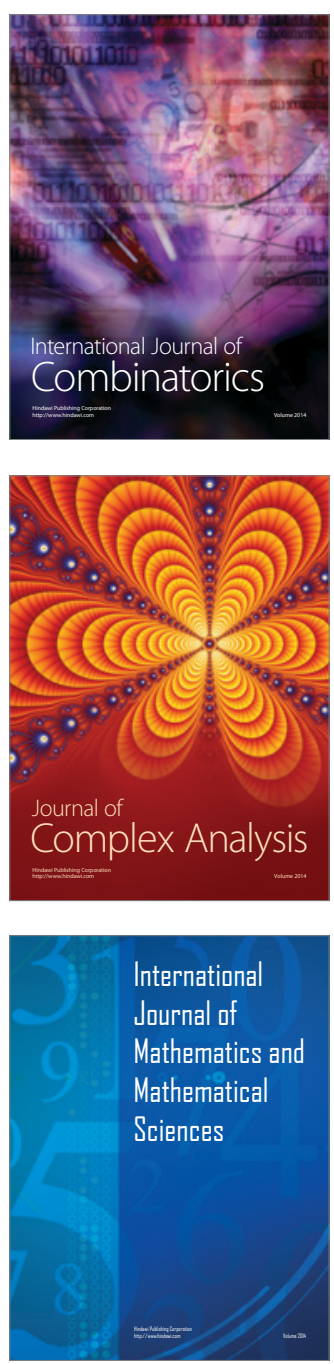
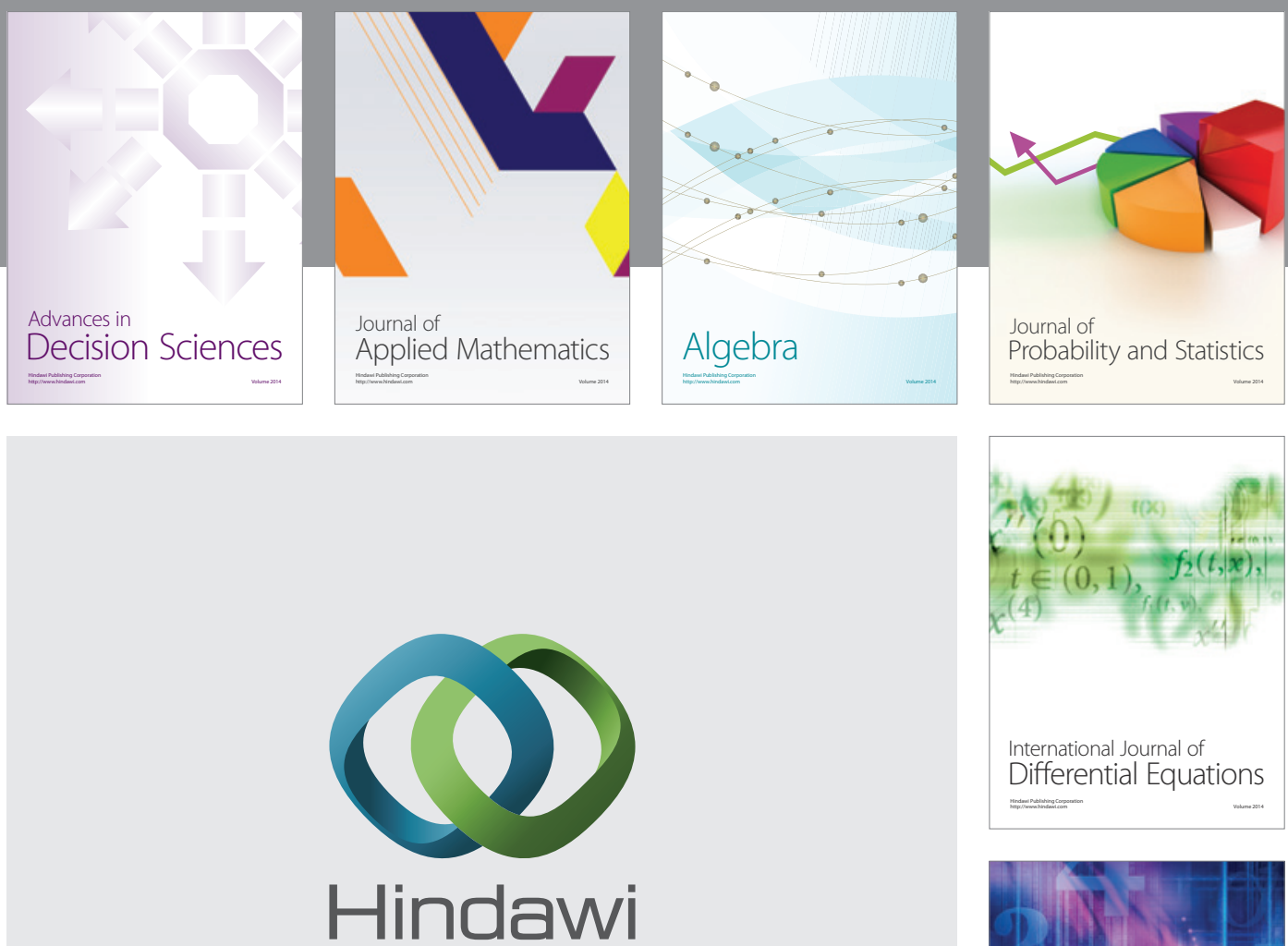

Submit your manuscripts at http://www.hindawi.com
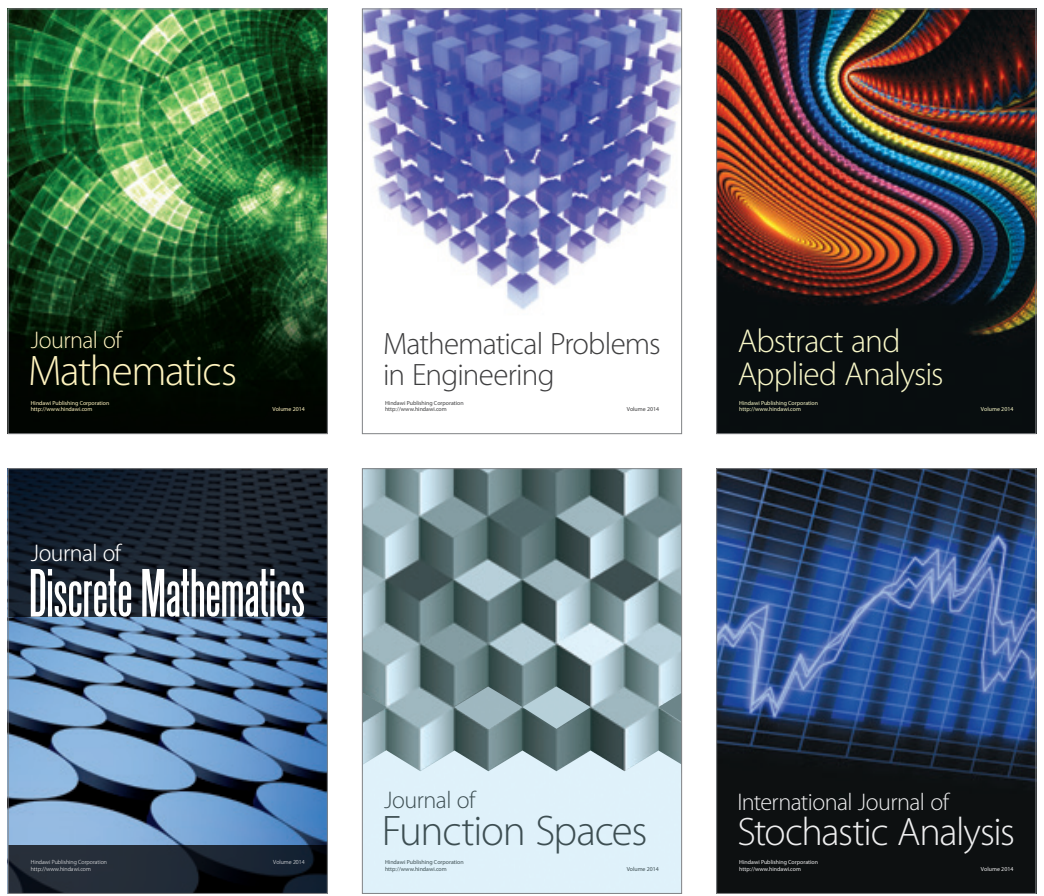

Journal of

Function Spaces

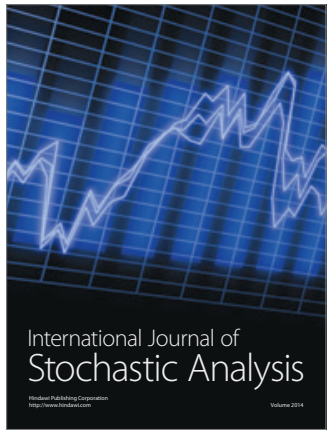

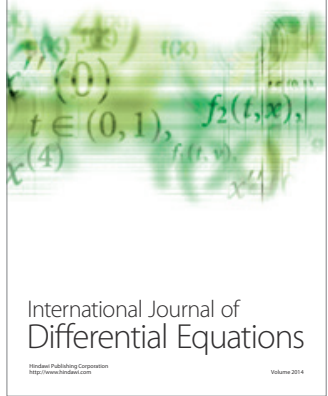
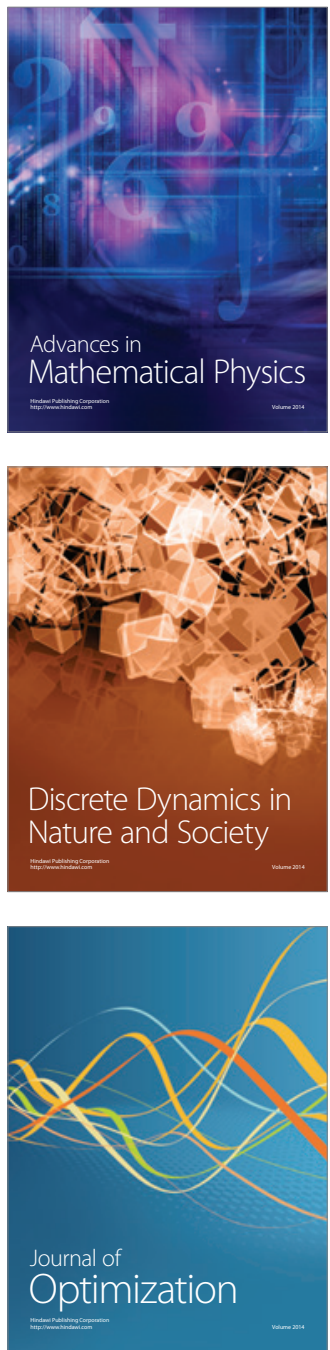